Article

\title{
An Energy-Aware Method for Selecting Cluster Heads in Wireless Sensor Networks
}

\author{
Atefeh Rahiminasab ${ }^{1}\left(\mathbb{D}\right.$, Peyman Tirandazi ${ }^{1, *}$ (D) M. J. Ebadi ${ }^{2}$, Ali Ahmadian ${ }^{3,4, *(\mathbb{D})}$ and \\ Mehdi Salimi ${ }^{5,6}$ \\ 1 Combinatorial and Geometric Algorithms Lab., Department of Mathematical Sciences, Yazd University, \\ Yazd 352, Iran; a.rahiminasab20@gmail.com or rahiminasab@stu.yazd.ac.ir \\ 2 Department of Mathematics, Chabahar Maritime University, Chabahar 545, Iran; ebadi@cmu.ac.ir \\ 3 Institute of IR 4.0, The National University of Malaysia, Bangi 43600 UKM, Malaysia \\ 4 School of Mathematical Sciences, Kean University (Wenzhou Campus), Wenzhou 325060, China \\ 5 Department of Mathematics \& Statistics, McMaster University, Hamilton, ON L8S 4K1, Canada; \\ mehdi.salimi@mcmaster.ca or mehdi.salimi@tu-dresden.de \\ 6 Center for Dynamics, Faculty of Mathematics, Technische Universität Dresden, 01062 Dresden, Germany \\ * Correspondence: ppp1486@gmail.com or tirandazy@stu.yazd.ac.ir (P.T.); ahmadian.hosseini@gmail.com or \\ aseyedal@kean.edu (A.A.)
}

Received: 19 September 2020; Accepted: 3 November 2020; Published: 6 November 2020

\begin{abstract}
Wireless sensor networks (WSNs) include several sensor nodes that have limited capabilities. The most critical restriction in WSNs is energy resources. Moreover, since each sensor node's energy resources cannot be recharged or replaced, it is inevitable to propose various methods for managing the energy resources. Furthermore, this procedure increases the network lifetime. In wireless sensor networks, the cluster head has a significant impact on system global scalability, energy efficiency, and lifetime. Furthermore, the cluster head is most important in combining, aggregating, and transferring data that are received from other cluster nodes. One of the substantial challenges in a cluster-based network is to choose a suitable cluster head. In this paper, to select an appropriate cluster head, we first model this problem by using multi-factor decision-making according to the four factors, including energy, mobility, distance to centre, and the length of data queues. Then, we use the Cluster Splitting Process (CSP) algorithm and the Analytical Hierarchy Process (AHP) method in order to provide a new method to solve this problem. These four factors are examined in our proposed approach, and our method is compared with the Base station Controlled Dynamic Clustering Protocol (BCDCP) algorithm. The simulation results show the proposed method in improving the network lifetime has better performance than the base station controlled dynamic clustering protocol algorithm. In our proposed method, the energy reduction is almost $5 \%$ more than the $\mathrm{BCDCP}$ method, and the packet loss rate in our proposed method is almost $25 \%$ lower than in the BCDCP method.
\end{abstract}

Keywords: wireless sensor networks; reduction of energy consumption; cluster head nodes; data aggregation; lifetime

\section{Introduction}

Wireless Sensor Networks (WSNs) consist of a large number of sensor nodes distributed randomly in a wide area to monitor the environment and allow the user to monitor the environment from a distant area. Moreover, WSNs are employed in numerous applications like military services, environmental monitoring [1,2], smart homes, medical applications [3], agriculture, and fire control [4]. Figure 1 illustrates the scheme of WSNs. Sensor nodes generally have sensing, processing, and communicating 
capabilities, and according to the structure, all have a distributed structure and measure the conditions of their surroundings. Furthermore, the primary task of sensor nodes is to gather the data at regular intervals and transform the information into an electronic signal. This signal is transmitted to the base station through a reliable wireless communication medium.

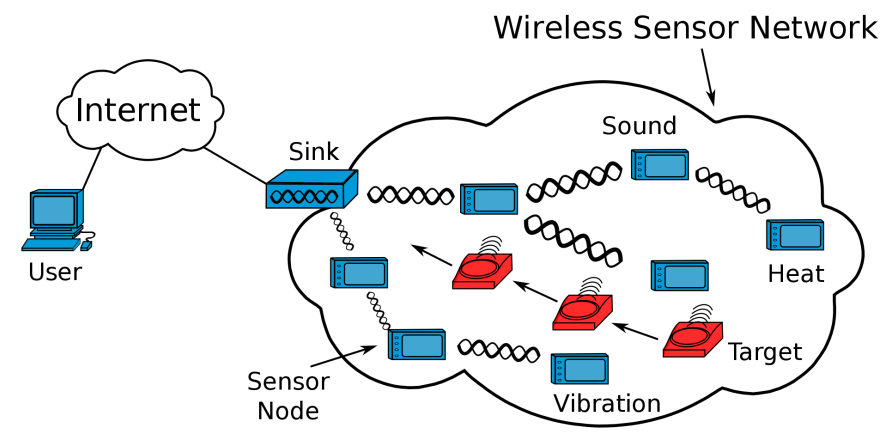

Figure 1. A sample of wireless sensor networks [1].

In early 1970, with the advent of microelectronics technology, new sensors were produced, which are inexpensive and have small dimensions and low weights. New raw materials for sensor construction were discovered and identified, and subsequently, a new procedure was introduced for collecting data. The integration of sensors and electronic signalling transformer circuits has provided significant opportunities for most applications [4].

Currently, although decreasing the size and weight and increasing the sensor sensitivity of sensor nodes are the primary goals of research laboratories and various companies, decreasing the size of sensor nodes leads to a reduction of the batteries' capabilities. The most important reason for the advent and development of WSNs is to monitor environments continuously where human accessibility and permanent presence is difficult or impossible. For instance, WSNs are used for monitoring the eruption of an active volcanic mountain, impassable border areas, and bridges. Consequently, recharging or replacing dead nodes is impossible because they are located in an inaccessible environment. As a result, due to the energy limitation of the nodes, lifetime and coverage are the main issues in WSNs' efficiency.

Since monitoring the applications requires much time, it is expected that sensor networks' lifetime is sufficiently long. Therefore, it is indispensable to divide an area into several sub-areas. By using this model, if several sensor nodes in each area die, others will almost maintain the network coverage. However, if all sensor nodes in an area die, monitoring the area becomes impossible, and network coverage will be destroyed. Consequently, to prolong the lifetime and maintain the network coverage in WSNs, the energy consumption of the nodes should be reduced, and this solution causes each node to consume energy uniformly.

Based on the solution mentioned above, the most significant goal in WSNs is energy consumption management. At first, it is critical to recognize how much energy is consumed in sensor nodes. Some of the energy consumption is beneficial, such as sending and receiving data, processing requested queries, and sending and receiving data queries to neighbour nodes. However, various activities in sensor nodes consume too much energy, as follows [5]:

1. Idle listening: Listening to inactive channels by several sensor nodes to receive possible traffic leads to idle listening.

2. Collision: The collision occurs when a sensor node receives more than one packet per time unit. All of the packets that lead to the collision should be resent, and this procedure increases energy consumption.

3. Eavesdropping: Eavesdropping happens when a sensor node receives packets that are sent to other nodes.

4. Overhead: Overhead is the minimum number of packets that should be used to send data. 
5. Unprepared node: An unprepared node is a node that is not ready to receive a message sent by other nodes.

It is indispensable to know how much energy is consumed in various parts of a sensor to manage energy consumption. Figure 2 shows the power consumption of sensor node subsystems. Energy consumption for the communication subsystem is higher than the computation subsystem. It is proved that sending 1-bit of information consumes energy as much as executing thousands of instructions [6], and the need to compromise between communication and processing is inevitable. Therefore, the most crucial issue is to decrease the energy consumption of sensor nodes.



Figure 2. Power consumption of sensor node subsystems [7].

\subsection{The Concept of Routing in Wireless Sensor Networks}

Routing is a considerable challenge due to several features in WSNs. The first feature is that there are many sensor nodes in WSNs. Based on this feature, it is impractical to build an addressing scheme for considerable sensor nodes because the ID maintenance overhead is too high. Therefore, the traditional IP-based protocol cannot be utilized in WSNs, and sensor nodes placed by the ad hoc method in the environment must be self-organized because sensor network operations need to be monitored. Consequently, these sensors require systems that establish communication and manage the distribution process. Moreover, in WSNs, received data are more critical than recognizing the ID of the data that should be sent. Second, unlike conventional communication networks, all WSN applications are required to transfer the sensed data from various sources to a specific base station. Third, sensor nodes have severe limitations in storage capability, energy, and processing. Thus, it is necessary to manage resources accurately. Forth, in most WSN scenarios, sensor nodes do not move after placing sensor nodes in the environment. By contrast, in a traditional wireless network, the sensor nodes can move everywhere, and the topology in this network changes constantly and unpredictably. However, in several applications, some sensor nodes may be able to move. Fifth, the requirements for the design of WSNs are determined by their applications. Sixth, for WSNs, it is indispensable to realize the status of sensor nodes because gathering data is based on their location, and GPS may provide this status. However, there are some methods by which we can find the location without GPS. Finally, gathering data in WSNs is performed by several sensor nodes. Therefore, the occurrence of redundancy in these data is tremendously high. This redundancy should be extracted by the routing protocol to increase the energy consumption and bandwidth. It is worth mentioning that WSNs are usually data-driven because data are requested according to several specific features. In the following, we express several considerable issues in the routing protocol:

1. Coverage: In WSNs, each sensor node can obtain some information about the area, and this information relies on the accuracy and range of sensor nodes. Furthermore, the information 
collected by the sensor node belongs to a specific part of the environment. Hence, area coverage is a significant issue that is used in WSNs' design.

2. Data aggregation: Since sensor nodes likely produce several redundant data, similar data packets generated from several nodes can be integrated to reduce the number of transfers. This method is used in several routing protocols to optimize energy consumption and data transfer.

3. Quality of Service (QoS): Data should be sent in several applications at a particular time; otherwise, the data will be useless. Therefore, the limited delay in data transfer is a condition for other time-consuming applications. On the other hand, in other applications, storing energy is more essential than the quality of service. However, to save energy in sensor nodes and increase the lifetime, it is indispensable to diminish the quality of the result. Thus, the routing protocol usually ignores the QoS.

\subsection{Routing Techniques in Wireless Sensor Networks}

According to the difference between WSNs and other networks, researchers have proposed various algorithms for the routing problem in WSNs. In these algorithms, authors have focused on WSNs' features and the network architecture. Furthermore, finding and maintaining a route is a considerable issue in WSNs because the energy restriction and considerable fluctuation in sensor nodes lead to frequent and unpredictable changes in the network's topological structure. Several proposed routing method use various methods including data aggregation, clustering, internal network processing, assigning a different role to the node, and data-based approaches to decrease energy consumption. We can generally classify all routing protocols into three categories, including flat, location-based, and hierarchical, according to the network structure. We state the hierarchical routing protocol, which is used in our study as below.

\section{Hierarchical Routing Protocol}

In this protocol, sensor nodes with a higher energy level are used to send and process the data. In contrast, the nodes with lower energy can sense the other sensor node that is in the vicinity of the target. In fact, in this protocol, we can enhance scalability, lifetime, and overall energy efficiency by creating clusters and assigning several tasks to the cluster head. Furthermore, merging and aggregating the data are performed to reduce the number of sent messages to the base station. Therefore, hierarchical routing is an effective protocol to reduce energy in a cluster. The hierarchical routing method is divided into two layers. The first layer is utilized to select an appropriate cluster head, and the second layer is used for the routing procedure. The hierarchical routing protocol is the most efficacious method to reduce energy consumption. Moreover, the Low-Energy Adaptive Clustering Hierarchy (LEACH), the Energy Efficient Clustering Scheme (EECS), and the Base station Controlled Dynamic Clustering Protocol (BCDCP) are the most important protocols, which belong to the hierarchical routing protocol.

In this paper, we aim to introduce a novel method to select an appropriate cluster head. We first model this problem by using multi-factor decision-making according to four factors. Then, we use the cluster splitting process and analytical hierarchy process algorithms to propose a novel method in order to solve this problem. The main contributions of this paper are as follows:

- All of the sensor nodes are prioritized according to four essential factors, including energy, mobility, distance to the centre, and the size of the data queue.

- The optimal number of cluster heads is computed, and the Cluster Splitting Process (CSP) algorithm is utilized to distribute the load on the cluster heads and cover all network nodes.

- The minimum spanning tree algorithm is utilized to distribute the energy load among the cluster heads.

- We consider the particular time to send the data from the sensor node to its cluster head. 
The organization of the paper is as follows. In Section 2, the related research works and previous techniques for cluster head selection are reviewed. We propose our method in Section 3. In Section 4, the effectiveness of our proposed method is evaluated. In Section 5, we express challenges and future work in WSNs. The conclusion are provided in Section 6.

\section{Literature Review}

Although authors have proposed several papers to select a suitable cluster head, we can categorize the methods into two groups. The first group has focused on the distribution of energy load with the cluster head selection. The authors in [8] proposed a cluster-based approach in which the energy load is distributed among the sensors in WSNs by using the rotation of local cluster-based stations, which is randomized. To improve robustness and scalability for dynamic networks, they used a coordination that was localized. Furthermore, for decreasing the amount of transmitted information to the base station, the data were also incorporated into the routing protocol. In [9], the authors developed an adaptive clustering hierarchy analysis with a low energy level. In this study, they proposed a protocol architecture for the networks that have microsensors. The authors also integrated the cluster-based routing and media access with each other. This method also consists of a new integrated cluster formation technique to save communication resources. By considering this method, the performance of the proposed algorithm considerably improves system lifetime, latency, and application-perceived quality.

In [10], the power consumption of wireless microsensor networks was reduced. The authors changed a communication protocol, and by using a deterministic component, they extended LEACH and proposed a new stochastic cluster head selection algorithm. Furthermore, to increase the lifetime of microsensor networks, they introduced a new method that uses three new metrics. In [11], the authors proposed a cluster head selection method in which the node degree is restricted. In order to reduce energy consumption and balance load, this method first chooses the cluster head nodes and then makes well-distributed clusters. Singha et al. [12] introduced particle swarm optimization to produce the clusters with an optimal cluster head selection. This method decreases the cost of placing the optimal position for the cluster head nodes. The authors in this study performed PSO implementation within the cluster, which forms a semi-distributed method. The objective function criteria were four parameters, including the residual energy, minimum average distance from the member nodes, and the headcount of the probable head nodes.

In [13], for heterogeneous WSNs, the authors proposed an improved distributed clustering scheme, which consumes energy efficiently and effectively. This method extends the lifetime and stability of the network. The authors in [14] proposed an energy efficient load balanced data gathering protocol. In this protocol, the partitioning procedure is performed by fuzzy logic, and the authors also used fuzzy logic to select an appropriate cluster head. This new approach was compared with LEACH, Fuzzy LEACH, and FCMLEACH in terms of prolonging the network lifetime and in a load balanced way. The performance of the proposed method is better than the other methods. Sarkar et al. [15] introduced a new cluster head method to transmit the data with the lowest energy consumed effectively. Furthermore, the node that is close enough to the base station and the other sensor is considered as a cluster head. For improving the energy consumption of the network, a new firefly algorithm was proposed. This algorithm also prolongs the lifetime of nodes by selecting the cluster head optimally.

The second group of methods, however, consider several significant factors to select an appropriate cluster head. Alghamdi et al. [16] proposed a new clustering model, which chooses the optimal cluster heads by considering four significant factors including energy, delay, distance, and security, and in this paper, the dragonfly and firefly algorithms were utilized to choose the optimal cluster heads.

In [17], the authors introduced an approach to select the cluster head according to the energy drain pattern and location of the sensor nodes. This method extends the lifetime of sensor nodes, and they expressed a variety of WSN probabilistic routing protocols to save energy during the data transmissions. Moreover, a mathematical model was proposed to increase the heterogeneity of the 
clustering algorithms. In order to reduce the energy drain in WSN nodes, the proposed method selects the cluster head according to the residual energy of sensor nodes.

Ngangbam et al. [18] introduced a new protocol to choose the optimal cluster heads to save battery energy. The method also extends the network lifetime by utilizing the limited power at the optimum level. In [19], two clustering routing protocols based on distance were proposed for WSNs. The cluster head selection method consists of solutions for two problems. The first problem is the proper distance from the candidate node to the base station, and the second problem is the remaining energy of a candidate node that wants to be a cluster head in the WSN. The performance of the two solutions was evaluated in terms of the number of dead nodes and energy consumption. The authors in [20] proposed a mathematical model that uses energy efficiently. They introduced the cluster head clustering protocol for mobile wireless sensor networks, which maximizes the network lifetime. This method includes the method of changing the cluster head in the cluster, which prevents repeated clustering and regularly distributes the load with the nodes. In [21-25], the authors proposed several creative methods to select a suitable cluster head by considering several factors.

The cluster routing method is the most efficient method to reduce energy consumption, which has been used in recent years. The most important protocol of this method is LEACH. Utilizing this method has led to developing another clustering method named the Base station controlled dynamic clustering protocol. This protocol is more efficient than other protocols in terms of increasing the lifetime. Therefore, in this paper, we use this protocol to propose an efficient approach for cluster head selection.

\section{Proposed Method}

Since the base station controlled dynamic clustering routing protocol is more efficient in extending the network lifetime than the other protocols mentioned previously, in the following, we describe this routing protocol in more detail [26].

\subsection{Base Station Controlled Dynamic Clustering Protocol}

In this protocol, it is obvious that selecting an inappropriate cluster head reduces the lifetime because by eliminating the cluster header, the connection between cluster member nodes with the network is disrupted. Thus, it is indispensable to choose a suitable approach to select an appropriate cluster head. The base station controlled dynamic clustering protocol in [26] is a network layer routing protocol and considers energy and distance as essential factors between sensor nodes in cluster heads. The base station plays a critical role in this protocol. Although the protocol, as LEACH, carries out the clustering process in the local phase, the main task of the base station controlled dynamic clustering protocol, such as clustering, routing, and random rotation of cluster heads, is carried out by the base station. Each round of this protocol has two phases, the installation and data transfer stages. In the installation phase, the base station receives the data about the energy level of all nodes, and those with a higher energy level than the average energy level are inserted into a set $(S)$. Cluster head nodes in the current round are selected from $S$. This selection ensures that nodes with sufficient energy are selected as cluster heads, and the other nodes with lower energy are used in other tasks. In this protocol, the base station utilizes the CSP algorithm to classify the nodes. In the transfer stage, however, the selected cluster heads collect data from the nodes, which belong to the particular cluster, and by using an optimal path determined by the base station, the data are transferred to the base station through the other cluster heads. After the end of each round, the base station forms a new set of cluster head nodes. There are three main reasons for which this protocol is more efficient than similar protocols. This protocol uses the balanced clustering technique to distribute equal load over all clusters. The CSP algorithm is utilized in each round of this protocol to select the cluster head with the maximum distance to the base station in order to distribute the cluster heads all over the environment. In this protocol, the most optimal route is used to transfer the data from the cluster head to the base station. 
The proposed method is based on the Base Station Controlled Dynamic Clustering Protocol (BCDCP). We use several key factors and the AHP method to select the most appropriate cluster heads. Moreover, computing the optimum number of cluster heads can enhance the BCDCP protocol. In fact, in this paper, we convert the cluster head selection problem into a multi-factor decision-making process. In this model, energy, mobility, distance to the centre, and the size of the data queue are considered as four essential factors. Then, we use the AHP and CSP algorithm to solve the problem.

The installation (creating clusters) and data transfer are the two stages in each round of the clustering algorithms. In the proposed method, we use the data transfer method proposed in [26]. Therefore, we describe the installation stage as follows:

1. Preliminaries:

1. The base station is located at a particular point, and it is far from the nodes of the network.

2. The primary energy of the sensor nodes is the same.

3. In the environment, there are several nodes with low mobility.

2. Energy model:

In order to assess the efficiency of the cluster maintenance in WSNs, we use the energy model in $[8,9]$ with new parameters. These parameters are listed in Table 1.

Table 1. Energy model used in the proposed method.

\begin{tabular}{ll}
\hline Amplifier energy & $\varepsilon_{\text {amp }}=100 \mathrm{pj} / \mathrm{bit} / \mathrm{m}^{2}$ \\
\hline Electronics energy & $E_{\text {elec }}=50 \mathrm{nj} / \mathrm{bit}$ \\
\hline $\begin{array}{l}\text { Communication energy parameters for the } \\
\text { multipath and free space model }\end{array}$ & $\varepsilon_{m p}=0.0013 \mathrm{pj} / \mathrm{bit} / \mathrm{m}^{4}, \varepsilon_{f s}=10 \mathrm{pj} / \mathrm{bit} / \mathrm{m}^{2}$ \\
\hline Energy for data aggregation & $E_{D A}=5 \mathrm{nj} / \mathrm{bit} / \mathrm{signal}$ \\
\hline
\end{tabular}

The energy to transmit a $k$ bit message up to distance $d$ :

$$
\begin{aligned}
E_{T X}(k, d)= & E_{\text {elec }}(k)+E_{\text {amp }}(k, d) \\
= & \begin{cases}k E_{\text {elec }}+k \varepsilon_{f s} \cdot d^{2}, & d<d_{0} \\
k E_{\text {elec }}+k \varepsilon_{m p} . d^{4}, & d \geq d_{0} \\
d_{0}=\sqrt{\frac{\varepsilon_{f s}}{\varepsilon_{m p}}} & \end{cases}
\end{aligned}
$$

The energy to receive a $k$ bit message:

$$
E_{R X}(k)=E_{\text {elec }}(k)=k \times E_{\text {elec }}
$$

Cluster head energy consumption with density coefficient $c$ and $n$ nodes:

$$
E_{C H}=n\left[E_{R X}(k)\right]+n\left[E_{D A}\right]+E_{T X}(\text { c.n.k.d })
$$

\subsection{Appropriate Criteria}

In our proposed method, we define four key factors, which play vital roles in extending the lifetime. Therefore, we consider four key factors to prioritize the sensor nodes for selecting an appropriate cluster head. We express these factors as follows:

1. Residual energy: Residual energy is the battery energy that remains in each node. Based on the primary energy definition, the energy consumption is calculated by Equations (1)-(3) in Table 1. 
2. Mobility: In order to decrease energy consumption, we consider mobility as an effective tool. The task of mobile nodes is to collect the data from static nodes, and the static nodes wait to receive data from the mobile nodes. It is worth mentioning that the connection between static nodes and mobile nodes occurs near each node. Consequently, static nodes save energy because the path length, the conflict between data that are being received or sent, and the overheads of sending data decrease severely.

3. Distance to the centre: This distance exemplifies the distance of each node from the centre of the network environment. We assume that the sensor nodes utilize GPS in order to determine the location.

4. Queue size: The queue size epitomizes the buffer capacity for each node. This parameter protects the data due to the huge amount of data in the receiver buffer.

It is indispensable to know the main task of the base station in the installation stage. Thus, we can categorize the main task of the base station into five groups as follows:

1. The base station considers the most appropriate nodes as cluster heads.

2. The base station calculates the optimum number of cluster heads.

3. The base station classifies the network nodes to reduce the total energy consumption in the data transfer phase.

4. The base station schedules transferring the data from non-cluster head nodes to the cluster heads.

5. The base station finds the most efficient path for the heads to communicate with each other and send data to the base station.

\subsection{Obtain a Set of Cluster Heads by Using the AHP Algorithm}

To organize and analyse complex decisions, we utilize the AHP method as a structured technique based on the mathematics model [27]. This method first decomposes the decision problem into a hierarchy of easier sub-problems, and we can analyse each of these sub-problems separately. Furthermore, according to each factor's importance, this method tries to find the best answers for the sub-problems. In this paper, we take advantage of the AHP method to select the most appropriate cluster head. In fact, AHP prioritizes the nodes to determine the highest sensor nodes' priority as the cluster head.

This process is performed in three phases: First of all, a hierarchical structure for the problem is designed. Second, the matrices are created, and the local load of factors or elements is calculated. Third, the results from the two previous steps are combined, and the total load of each node is calculated. In the following, we express these steps as follows:

Step 1:

According to Figure 3, the structure consists of goals, decision-making factors, and nodes. The first level is our goal, which is to select the most appropriate cluster head. The second level shows the decision-making factors, and the third level illustrates the nodes. 


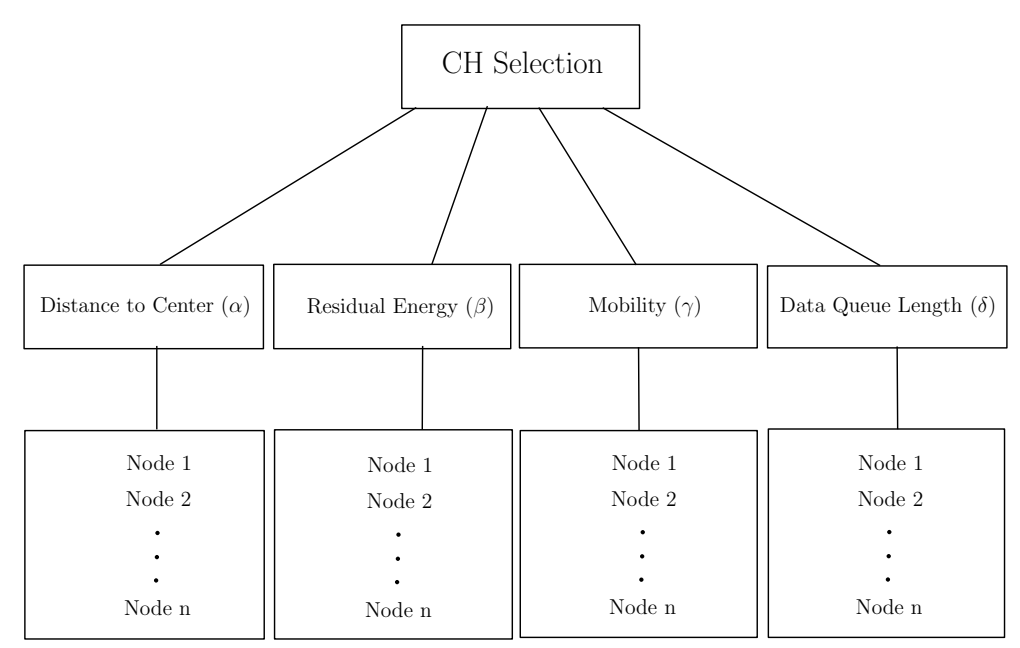

Figure 3. The structure of AHP for cluster head selection.

\section{Step 2:}

The local load includes the load of each factor relative to the goal and the load of each candidate node relative to any factor for which both parts can be calculated in the same way. In fact, it is shown by the formation of matrices which factor/element is more important and by how much. Table 2 [25] illustrates a fundamental scale, ranked from 1-9. To compare the pair to pair of four factors, a $4 \times 4$ matrix is created where $a_{i}$ is the ratio of the factor load of $i$ to the factor of load of $j$.

$$
A=\left[\begin{array}{rrrr}
a_{\frac{\alpha}{\alpha}} & a_{\frac{\alpha}{\beta}} & a_{\frac{\alpha}{\gamma}} & a_{\frac{\alpha}{\delta}} \\
a_{\frac{\beta}{\alpha}} & a_{\frac{\beta}{\beta}} & a_{\frac{\beta}{\gamma}} & a_{\frac{\beta}{\delta}} \\
a_{\frac{\gamma}{\alpha}} & a_{\frac{\gamma}{\beta}} & a_{\frac{\gamma}{\gamma}} & a_{\frac{\gamma}{\delta}} \\
a_{\frac{\delta}{\alpha}} & a_{\frac{\delta}{\beta}} & a_{\frac{\delta}{\gamma}} & a_{\frac{\delta}{\delta}}
\end{array}\right]
$$

$A W=\lambda_{\max }$ is calculated for matrix $A$ in which $\lambda$ is the eigenvalue and $W$ is its corresponding nonzero eigenvector. Moreover, the local load of each factor is computed by $W^{T}$, and the eigenvector is as Equation (4).

$$
W^{T}=\left\{W_{\alpha}, W_{\beta}, W_{\gamma}, W_{\delta}\right\}
$$

If for each matrix element, we consider $a_{\frac{i}{j}}=\frac{1}{a_{j}}$ and $a_{\frac{i}{j}}=a_{\frac{k}{j}} \times a_{\frac{i}{k}}$, matrix $A$ will be coherent. Moreover, unfortunately, due to random judgements of individuals, evaluation matrices are usually not fully consistent. Therefore, errors in judgements can be detected by a consistency ratio $(C R)$, which is defined as the ratio of the consistency index $(C I)$ to the random index $(R I)$. CI can be calculated as follows:

$$
\begin{aligned}
C I & =\frac{\left(\lambda_{\max }-n\right)}{n-1} \\
\lambda_{\max } & =\left(\frac{1}{n}\right) \sum_{i=1}^{n} \frac{(A W)_{i}}{W_{i}},
\end{aligned}
$$


Table 2. A fundamental scale (one to nine). The value of 2, 4, 6, and 8 is considered as the average value between the number before and after them.

\begin{tabular}{cc}
\hline Number Rating & Rank \\
\hline Equally & 1 \\
Moderately & 3 \\
Strongly & 5 \\
Very Strongly & 7 \\
Extremely Strongly & 9 \\
\hline
\end{tabular}

Table 3 [25] illustrates the random index $(R I)$. Based on these calculation, if $C R \leq 0.1$, the difference between opinions is almost good, and $W$ can be considered as the corresponding component load. Otherwise, the elements of matrix A should be adjusted until matrix A satisfies desirable consistency.

Table 3. Random index.

\begin{tabular}{ccccccccc}
\hline Degree & $\mathbf{1 , 2}$ & $\mathbf{3}$ & $\mathbf{4}$ & $\mathbf{5}$ & $\mathbf{6}$ & $\mathbf{7}$ & $\mathbf{8}$ & $\mathbf{9}$ \\
\hline Random Index & 0 & 0.58 & 0.9 & 1.12 & 1.24 & 1.32 & 1.41 & 1.45 \\
\hline
\end{tabular}




\section{Step 3:}

By using the above steps, not only a load of each decision factor $\left(w_{j}\right)$, but the load of sensor nodes $\left(w_{n_{i}}\right)$ can be calculated. We assume that five sensor nodes are in the wireless sensor network. Thus, the node load matrix can be created as follows:

$$
A=\left[\begin{array}{cccc}
W_{\frac{n_{1}}{\alpha}} & W_{\frac{n_{1}}{\beta}} & W_{\frac{n_{1}}{\gamma}} & W_{\frac{n_{1}}{\delta}} \\
W_{\frac{n_{2}}{\alpha}} & W_{\frac{n_{2}}{\beta}} & W_{\frac{n_{2}}{\gamma}} & W_{\frac{n_{2}}{\delta}} \\
W_{\frac{n_{3}}{\alpha}} & W_{\frac{n_{3}}{\beta}} & W_{\frac{n_{3}}{\gamma}} & W_{\frac{n_{3}}{\delta}} \\
W_{\frac{n_{4}}{\alpha}} & W_{\frac{n_{4}}{\beta}} & W_{\frac{n_{4}}{\gamma}} & W_{\frac{n_{4}}{\delta}} \\
W_{\frac{n_{5}}{\alpha}} & W_{\frac{n_{5}}{\beta}} & W_{\frac{n_{5}}{\gamma}} & W_{\frac{n_{5}}{\delta}}
\end{array}\right]
$$

Therefore, the overall load matrix is calculated as follows:

$$
W_{n_{i}}=W_{\frac{n_{i}}{j}} \times W_{j}
$$

Finally, the overall load of each node can be computed as follows:

$$
W_{n_{i}}=\sum_{j=1}^{4} W_{\frac{n_{i}}{j}} \times W_{j}
$$

The nodes with the greatest load have the highest priority, a certain number of which $(k)$ form a set of cluster heads in the current round.

\subsection{Calculate the Optimal Number of Cluster Heads $(k)$}

In this paper, we use the simulated annealing algorithm to calculate the optimal number of cluster heads [28]. This algorithm reduces the energy consumption of nodes that transfer their data to cluster heads and does this by reducing the total distance between the nodes and closest cluster heads. We suppose that $N$ nodes are in the environment. If the number of clusters is $k$, each cluster has $\frac{N}{k}$ nodes (one cluster and $\frac{N}{k}-1$ nodes).

As mentioned previously, the task of cluster heads is to send the integrated data to the base station, and the base station is far away from the nodes. Therefore, wasted energy is calculated by the multipath model. Consequently, cluster heads' energy consumption for a signal frame is calculated as follows:

$$
E_{C H}=l \times E_{\text {elec }}\left(\frac{N}{k}-l\right)+l \times E_{D A} \times\left(\frac{N}{k}\right)+l \times E_{\text {elec }}+l \times \epsilon_{m p} d_{\text {toBS }}^{4}
$$

In Equation (10), $l$ is the number of data bits, and $d_{t o B S}$ is the distance between the cluster head and base station.

Each node sends its data to the cluster head. Hence, the energy consumption is calculated by the FRIISfree space model as follows:

$$
E_{\text {non }-\mathrm{CH}}=l \times E_{\text {elec }}+l \times \epsilon_{f s} \times d_{\text {toCH }}^{2}
$$


We assume that nodes are distributed in an $M \times M$ environment, and the area for each cluster is almost $\frac{M^{2}}{k}$. Furthermore, the cluster head is in the centre of the cluster, and the nodes' distance from the cluster head is computed as follows:

$$
d_{t o C H}^{2}=\iint\left(x^{2}+y^{2}\right) \rho(x, y) d x d y=\iint r^{2} \rho(r, \theta) r d r d \theta
$$

If the area is the circle with radius $R=\left(\frac{M}{\sqrt{\pi \times k}}\right)$, Equation (12) will be changed as Equation (13):

$$
d_{\text {toCH }}^{2}=\rho \int_{0}^{2 \pi} \int_{0}^{\frac{M}{\sqrt{\pi k}}} r^{3} d r d \theta=\frac{\rho}{2 \pi} \times \frac{M^{4}}{k^{2}}
$$

If the density of nodes in the cluster is the same, then $\rho=\frac{1}{\frac{M^{2}}{k}}$, and we will have Equation (14).

$$
d_{t o C H}^{2}=\frac{1}{2 \pi} \times \frac{M^{2}}{k}
$$

Therefore, we have Equation (11) as follows:

$$
E_{n o n-C H}=l \times E_{e l e c}+l \times \epsilon_{f s} \times \frac{1}{2 \pi} \times \frac{M^{2}}{k}
$$

Energy consumption for each cluster is computed as follows:

$$
\begin{aligned}
E_{\text {cluster }} & =E_{C H}+\left(\frac{N}{k}-1\right) \times E_{\text {non-CH }} \\
& \approx E_{C H}+\left(\frac{N}{k}\right) \times E_{\text {non }-C H}
\end{aligned}
$$

Overall energy consumption per each frame is computed as follows:

$$
\begin{aligned}
E_{\text {total }} & =k \times E_{\text {cluster }} \\
& =l\left(2 \times E_{\text {elec }} \times N+E_{D A} \times N+k \times \epsilon_{m p} \times d_{\text {toBS }}^{4}+E_{\text {elec }} \times N+\epsilon_{f_{s}} \times \frac{1}{2 \pi} \times \frac{M^{2}}{k} \times N\right)
\end{aligned}
$$

Finally, the optimum cluster head will be computed as follows:

$$
k=\frac{\sqrt{N}}{\sqrt{2 \pi}} \times \frac{\sqrt{s f^{\epsilon}}}{\sqrt{p m^{\epsilon}}} \times \frac{M}{d_{\text {toBS }}^{2}}
$$

\subsection{Running the CSP Algorithm on a Set of Cluster Heads}

In this section, the CSP algorithm runs on a set of cluster heads. First of all, the network is divided into two distinct clusters. Then, each cluster is divided into smaller clusters. This process will be continued since the number of clusters $(k)$ is obtained.

In each phase of the CSP algorithm, this algorithm selects the cluster head that has the farthest distance from other cluster heads. This selection ensures the coverage of all of the nodes in the network [26]. Furthermore, for the same load distribution on the cluster head, the clustering technique in [29] is used. Based on this technique, each cluster is decomposed until the number of achieved cluster heads is approximately equal. Therefore, the four steps of this algorithm are described as follows: 
1. In cluster head sets, two nodes are selected with the farthest distance from each other.

2. Based on the distance, all remaining nodes (other members of cluster heads and nodes) from two selected cluster heads are located in a closer cluster head.

3. The two cluster head sets are the same when they have approximately the same number of nodes. As a result, two sub-clusters are formed.

4. According to Step 3, cluster head sets are divided into smaller sets.

After categorizing sensor nodes and selecting the cluster heads, one of the most important issues that should be taken into consideration is to establish the best connection between cluster heads for transferring data to the base station. Sending data to base stations consumes much energy, whereas, in normal conditions, the nearest cluster head is always selected to transfer data. Thus, as shown in Figure 4, the energy load can be divided by the random selection of the cluster head and the minimum spanning tree [30]. Finally, the base station determines a scheduled program for other nodes to send the data to their cluster head.

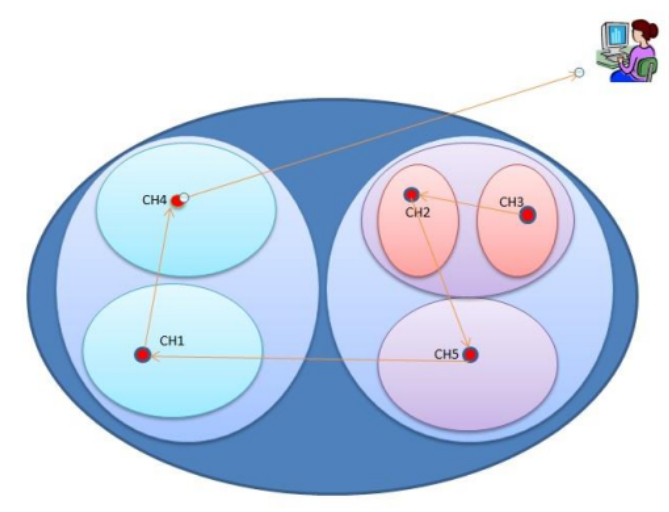

Figure 4. An overview of the Cluster Splitting Process (CSP) algorithm.

\section{Simulations and Results}

In this part, in order to evaluate the performance of the proposed method, our method is compared with the BCDCP cluster-based protocol. It is worth mentioning that all of the experiments were performed 50 times on an Intel Core i7 CPU with 16.00 GB RAM running on Windows 10 implemented in MATLAB 2020a. In the following, first of all, we state the input parameters that are essential for the simulation.

In our experiment, we considered 100 nodes in WSNs, where each node is assigned an initial energy of $0-2$ joules and randomly distributed in a $100 \times 100$ square domain. In addition, the capacity of the buffer for each node is five packets, while the length of each packet is 512 bits, and the sending rate is $9.6 \mathrm{~Kb} / \mathrm{s}$. Furthermore, The location of the base station is $(200,100)$. Moreover, the mobility of the nodes is one of these modes, including $0 \mathrm{~m} / \mathrm{s}, 1 \mathrm{~m} / \mathrm{s}$, or $3 \mathrm{~m} / \mathrm{s}$, and the direction of the nodes are considered random. In the AHP algorithm, according to the equations in Section 3.3, we have:

$$
A=\left[\begin{array}{cccc}
1 & \frac{1}{2} & \frac{3}{2} & 3 \\
2 & 1 & 3 & 4 \\
\frac{2}{3} & \frac{1}{3} & 1 & 2 \\
\frac{1}{3} & \frac{1}{4} & \frac{1}{2} & 1
\end{array}\right]
$$


The eigenvector is as below:

$$
W^{T}=\{0.291,0.486,0.121,0.101\}
$$

Each local load of the factors determines the distance to the centre, residual energy, nodes' mobility, and data queue length. It is clear that residual energy has the most priority, and data queue length has the least priority. We can calculate the eigenvalue and consistency ratio based on Equations (5) and (6). Therefore, the eigenvalue and consistency ratio are $\lambda_{\max }=4.1575$ and $C R=0.0058<0.1$, respectively. Thus, matrix A is coherent. Similarly, four $100 \times 100$ matrices are created to compare pairwise all network nodes based on the four factors that we mentioned previously, and the eigenvector $(4 \times 100$ matrix) based on Equation (20) presents the load of each node for each factor. As a result, the total load based on Equations (8) and (9) is created, and all nodes are prioritized based on the degree of importance and whether each node can be a cluster head or not.

$$
W_{\text {Total }}^{T}=W \times W_{n_{i}}=\left[S_{1}, S_{2}, S_{3}, S_{4}, \ldots, S_{100}\right]
$$

where $S_{i}$ is the priority of sensor $i$ to be the cluster head. Therefore, if $S_{i}$ has a greater value, the priority of sensor $i$ is higher than the other sensors. Based on Equation (18), the optimal number of cluster heads is five $(k=5)$, and we also consider this number for the first round of experiment. Thus, the base station selects the five nodes with the highest priority based on Equation (20), and the CSP algorithm is performed by utilizing these five nodes.

Since we distribute the nodes randomly in the environment, the location of the nodes is different. Therefore, we execute the algorithm 50 times.

In the first experiment, we compare the proposed method (AHP-CSP) and BCDCP by the number of alive nodes that remain in the network at $1600 \mathrm{rpm}$. Figure 5 illustrates the number of alive nodes, and as we can see, in 1010 rounds, all nodes in our proposed method are alive. However, the first node in BCDCP loses in 954 rounds. If we consider the network lifetime as the number of alive nodes, therefore, the network lifetime in our proposed method is more effective than BCDCP.

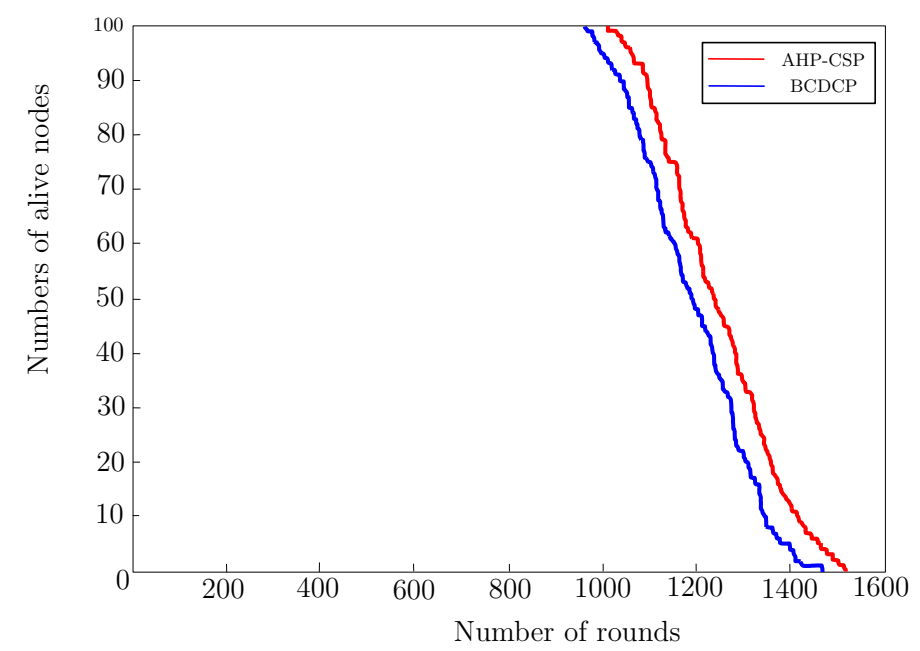

Figure 5. A comparison between AHP-CSP and the Base station Controlled Dynamic Clustering Protocol (BCDCP) by the number of alive nodes (system lifetime).

In the second experiment, the total number of received messages by the base station was examined in 1600 rounds. As shown in Figure 6, the maximum number of messages in our proposed method is received by the base station in the 1000th round (12,510 messages). Moreover, when the number of active nodes reduces, the received data also decrease. This process can be observed for the BCDCP method, but the maximum number of received messages by the base station occurs in the 954th round $(12,100$ messages). Therefore, the impact on the received message increase in our proposed method in 
comparison with the BCDCP method is considerable. However, it is indispensable to know, when we send data packets to the base station, what the status of the energy levels of the nodes is.

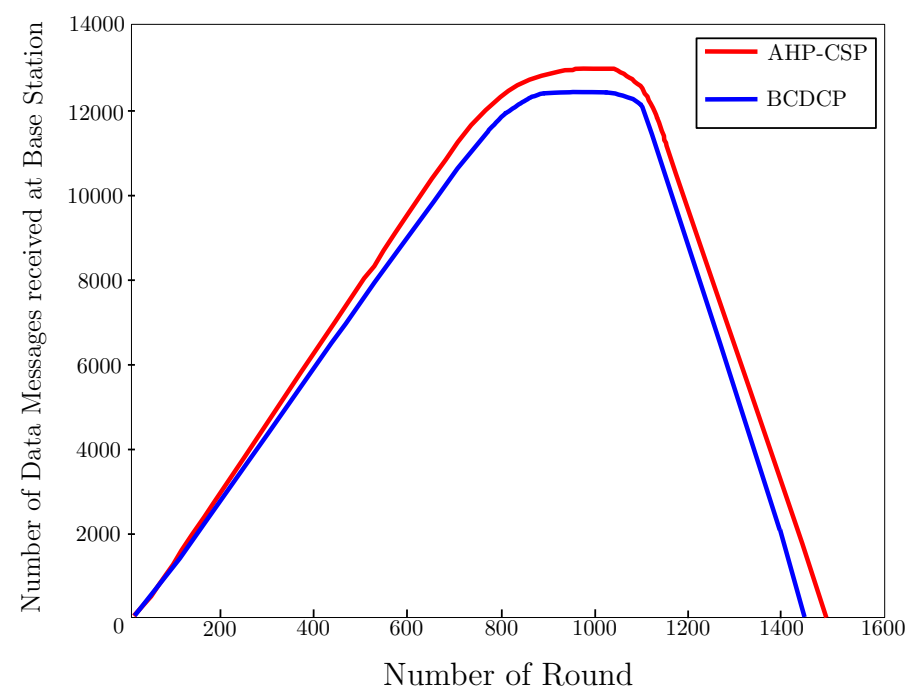

Figure 6. Total number of data messages received at the base station according to the number of operation rounds.

As we mentioned previously, the initial energy of all nodes in the network is at most 2J. Figure 7 presents the number of transferred messages per energy unit. The number of transferred messages per energy unit to the base station in our proposed method is greater than the BCDCP method.

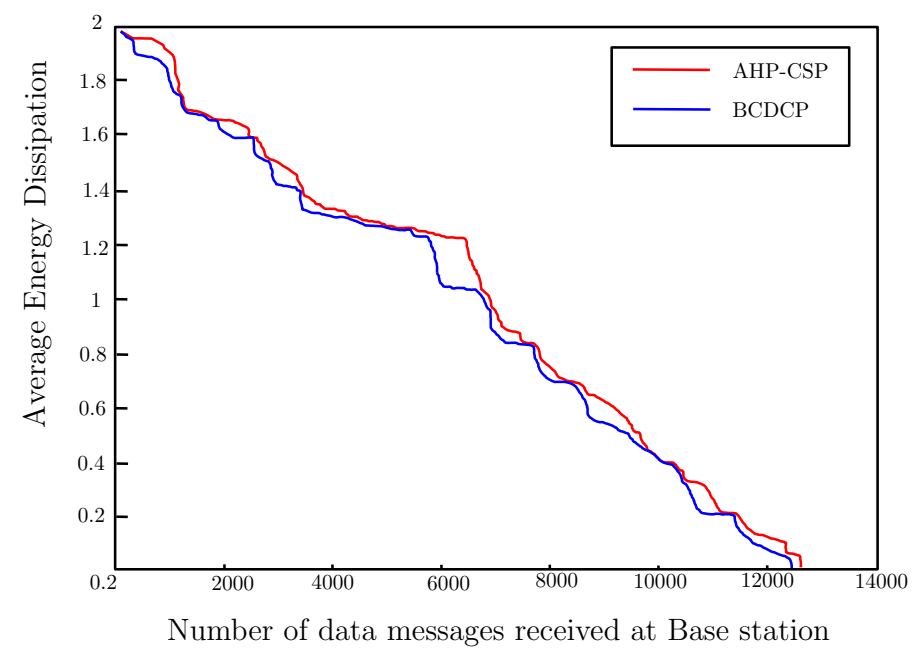

Figure 7. The total number of data messages received at the base station as a function of average energy dissipation.

As shown in Figure 8, the energy consumption in the proposed method (AHP-CSP) is less than the BCDCP method. In both methods, we use the cluster head routing method, and the only task of one cluster head is to send data to the base station. In the proposed algorithm, two critical factors in cluster head selection, distance to the centre and mobility, lead to the energy reduction, and in our proposed method, the energy reduction is almost $5 \%$ more than the BCDCP method.

In Figure 9, the increase of the average energy consumption according to time is compared. As shown in Figure 9, the average lost energy in the BCDCP method is more than our proposed method. An appropriate cluster head selection and the data transfer load balance between nodes lead to uniform energy consumption. 


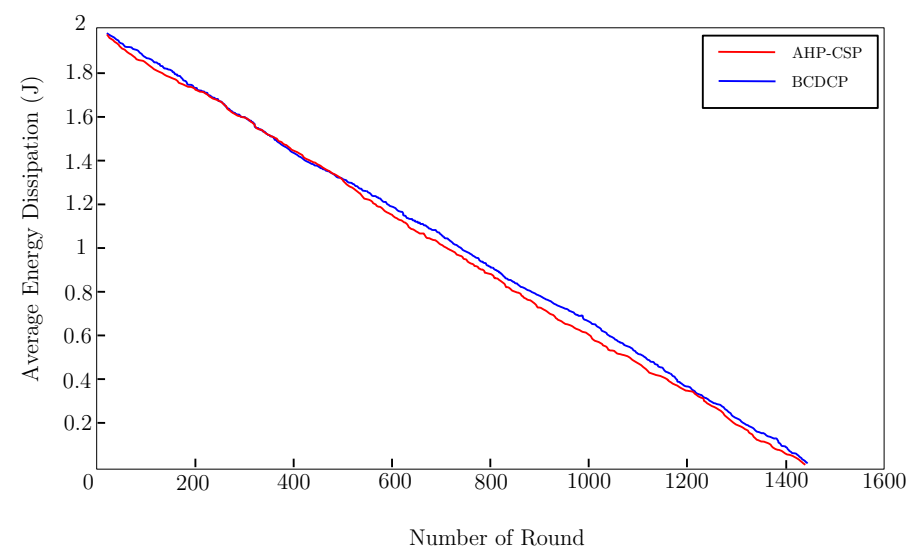

Figure 8. A comparison between AHP-CSP and BCDCP in terms of average energy dissipation.



Figure 9. A comparison between AHP-CSP and BCDCP in terms of average lost energy.

Finally, in Figure 10, we compare the two methods in terms of the packet loss rate. As we know, data packets may be lost in two ways, insufficient capacity of the buffer in receive node and insufficient energy for packet transfer. Figure 10 shows that due to residual energy and buffer capacity, the packet loss rate in our proposed method is almost $25 \%$ lower than in the BCDCP method.



Figure 10. A comparison between AHP-CSP and BCDCP in terms of the packet loss rate. 


\section{Challenges and Future Work}

Given the advent of wireless sensor networks and the recent growth in the methods of wireless sensor networks during the last decade, authors have proposed creative methods to meet the existing challenges. However, several challenges can be discussed for future work as stated in the following [31].

Time is a critical challenge in wireless sensor networks. WSNs deal with a real-world environment. In many cases, sensor data must be delivered to the base station under time constraints so that proper observations are made or immediate actions are taken. To date, few studies have been done to meet the real-time requirements in WSNs. Not only do most protocols overlook the real-time requirements, they also attempt to take prompt actions that are fast enough to meet the deadline. There are some initial findings regarding real-time routing. Some other functions of data transmission, data fusion, classification, security, query processing, target, and event detection must also face real-time limitations. Developing real-time protocols for WSNs, as well as the associated analysis techniques is important.

Another challenge for WSNs is power management or the energy constraint. The slow growth in battery capacity development and the inhibition of battery replacement by the untended nature of sensor nodes and the perilous sensing environment lead to some difficulties in facing this constraint. Furthermore, the vigilant nature of sensor network applications entails a long lifetime. On the other hand, finding an energy effective surveillance service for a geographic region is of great importance. Therefore, the focus of current research is on the ways of providing either full or partial sensing coverage within the context of energy conservation.

The next challenges can be the time-varying nature and the network scale in WSNs. Sensor nodes function with limited capabilities of communication, storage, and computing under severe constraints on energy. Depending on the application, there are varying densities of WSNs ranging from sparse to highly dense. The sensor nodes show highly adaptive and dynamic behaviour. The need to be self-organized and conserve energy make sensor data constantly adjust their behaviour to the current level of activity. Furthermore, the behaviour of sensor nodes may need to be adjusted in response to the haphazard and unpredictable behaviour of wireless connections arising from the high level of noises and interference in the radio frequency. This adjustment is for inhibiting severe degradation of the performance of the supported application.

The last challenge may be distance management. Sensor nodes may be implemented in other fields such as subway stations. The direct management of the network will be difficult, and thus, an indirect management or distant control system is needed.

\section{Conclusions}

In this paper, we propose a new centralized clustering algorithm in which the base station does not have energy limitations, as an important component of the network. The main objective is to reduce the energy consumption and enhance the performance of the packet loss rate. To reduce energy consumption, we use a multi-factor decision-making (energy, mobility, distance to the centre, and the length of data queues) and two familiar methods, including the Analytical Hierarchy Process (AHP) and the Cluster Splitting Process (CSP). These two methods perform various tasks, including selecting cluster heads, computing the optimum number of cluster heads, identifying the optimal path between cluster heads, and scheduling for sending data from nodes to the cluster heads. We compare our proposed method with the Base station Controlled Dynamic Clustering Protocol (BCDCP) in terms of increasing the network lifetime. The energy reduction is almost $5 \%$ more than the BCDCP method, and the packet loss rate in our proposed method is almost $25 \%$ lower than in the BCDCP method. Consequently, the results show that the proposed method in improving the network lifetime has better performance than the BCDCP method.

Author Contributions: All authors have read and agreed to the published version of the manuscript.

Funding: This research received no external funding.

Conflicts of Interest: The authors declare no conflict of interest. 


\section{References}

1. Al-Turjman, F.M.; Hassanein, H.S.; Ibnkahla, M. Towards prolonged lifetime for deployed WSNs in outdoor environment monitoring. Ad Hoc Netw. 2015, 24, 172-185. [CrossRef]

2. Paek, J.; Hicks, J.; Coe, S.; Govindan, R. Image-Based Environmental Monitoring Sensor Application Using an Embedded Wireless Sensor Network. Sensors 2014, 14, 15981-16002. [CrossRef] [PubMed]

3. Zhongwei, Z. IEEE 802.14. 5/ZigBee based WSNs-WPANs and innovative applicationin medical health care systems. Stud. Health Technol. Inform. 2014, 207, 125-134.

4. Saravanan, A.; Prasanna, V.; Malathi, S.; Balaji, M. Cracker Industry Fire Monitoring System Over Cluster Based WSN. J. Eng. Appl. Sci. 2014, 9, 1-5.

5. Anastasi, G.; Conti, M.; Gregori, E.; Passarella, A. 802.11 power-saving mode for mobile computing in Wi-Fi hotspots: Limitations, enhancements and open issues. Wirel. Netw. 2008, 14, 745-768. [CrossRef]

6. Akyildiz, I.F.; Su, W.; Sankarasubramaniam, Y.; Cayirci, E. A survey on sensor networks. IEEE Commun. Mag. Wirel. Netw. 2002, 40, 102-114. [CrossRef]

7. Park, G.; Rosing, T.; Todd, M.D.; Farrar, C.R.; Hodgkiss, W. Energy harvesting for structural health monitoring sensor networks. J. Infrastruct. Syst. 2008, 14, 64-79. [CrossRef]

8. Heinzelman, W.R.; Chandrakasan, A.; Balakrishnan, H. Energy-efficient communication protocol for wireless microsensor networks. In Proceedings of the 33rd Annual Hawaii International Conference on System Sciences, Maui, HI, USA, 7 January 2000; Volume 43, pp. 3005-3014.

9. Heinzelman, W.R.; Chandrakasan, A.; Balakrishnan, H. An application-specific protocol architecture for wireless microsensor networks. IEEE Trans. Wirel. Commun. 2002, 1, 660-670. [CrossRef]

10. Handy, M.J.; Haase, M.; Timmermann, D. Low energy adaptive clustering hierarchy with deterministic cluster head selection. In Proceedings of the 4th International Workshop on Mobile and Wireless Communications Network, Stockholm, Sweden, 9-11 September 2002; Voume 1, pp. 368-372.

11. Mahajan, S.; Malhotra, J.; Sharma, S. An energy balanced QoS based cluster head selection strategy for WSN. Egypt. Inform. J. 2014, 15, 189-199. [CrossRef]

12. Singha, B.; Lobiyal, D.K. Energy-aware cluster head selection using particle swarm optimization and analysis of packet retransmissions in WSN. Procedia Technol. 2012, 4, 171-176. [CrossRef]

13. Saini, P.; Sharma, A.K. E-DEEC-Enhanced Distributed Energy Efficient Clustering Scheme for heterogeneous WSN. In Proceedings of the 1st International Conference on Parallel, Distributed and Grid Computing, Solan, India, 28-30 October 2010; pp. 205-210.

14. Mondal1, S.; Dutta, P.; Ghosh1, S.; Biswas, U. Energy Efficient Rough Fuzzy Set based Clustering and Cluster-Head Selection for WSN. In Proceedings of the 2nd International Conference on Next Generation Computing Technologies, Dehradun, India, 14-16 October 2016; pp. 439-444.

15. Sarkar, A.; Murugan, T.S. Cluster-head selection for energy efficient and delay-less routing in wireless sensor network. Wirel. Netw. 2019, 25, 303-320. [CrossRef]

16. Alghamdi, T.A. Energy efficient protocol in wireless sensor network: Optimized cluster head selection model. Telecommun. Syst. 2020, 74, 331-345. [CrossRef]

17. Koyuncu, H.; Tomar, G.S.; Sharma, D. A New Energy Efficient Multitier Deterministic Energy-Efficient Clustering Routing Protocol for Wireless Sensor Networks. Symmetry 2020, 12, 837. [CrossRef]

18. Ngangbam, R.; Hossain, A.; Shukla, A. Improved low energy adaptive clustering hierarchy and its optimum cluster head selection. Int. J. Electron. 2019, 107, 1-13. [CrossRef]

19. Nguyen, T.G.; Soin, C.; Nguyen, N.G. Two Energy-Efficient Cluster-head Selection Techniques Based on Distance for Wireless Sensor Networks. In Proceedings of the 2014 International Computer Science and Engineering Conference, Khon Kaen, Thailand, 30 July-1 August 2014; pp. 33-38.

20. Rajesh, D.; Jaya, T. A Mathematical Model for Energy Efficient Secured CH Clustering Protocol for Mobile Wireless Sensor Network. Wirel. Pers. Commun. 2020, 112, 421-438. [CrossRef]

21. Chatterjee, M.; Das, S.K.; Turgut, D. An on-demand weighted clustering algorithm (WCA)for ad hoc networks. In Proceedings of the Globecom '00-IEEE. Global Telecommunications Conference, Conference Record (Cat. No. 00CH37137), San Francisco, CA, USA, 27 November-1 December 2000; Volume 3, pp. 1697-1701.

22. Gupta, I.; Riordan, D.; Sampalli, S. Cluster-head selection using Fuzzy Logic for wireless sensor network. In Proceedings of the 3rd Annual Communication Networks and Services Research Conference, Halifax, NS, Canada, 16-18 May 2005; pp. 255-260. 
23. Liang, Q. Clusterhead election for mobile ad hoc wireless network. In Proceedings of the 14th IEEE Proceedings on Personal, Indoor and Mobile Radio Communications, PIMRC 2003, Beijing, China, 7-10 September 2003; pp. 1623-1628.

24. Tillapat, P.; Thumthawatworn, T.; Pakdeepinit, P.; Yeophantong, T.; Charoenvikrom, S.; Daengdej, J. Method for cluster heads selection in wireless sensor networks. In Proceedings of the IEEE Aerospace Conference, Big Sky, MT, USA, 6-13 March 2004; Volume 6, pp. 3615-3623.

25. Yin, Y.; Shi, J.; Li, Y.; Zhang, P. Cluster-head selection using Analytical Hierarchy Process for wireless sensor networks. In Proceedings of the 17th Annual IEEE International Symposium on Personal, Indoor and Mobile Radio Communications, Helsinki, Finland, 11-14 September 2006; pp. 1-5.

26. Muruganathan, S.D.; Daniel, C.F.M.; Bhasin, R.I.; Fapojuwo, A. A Centraized Energy-Efficient Rouized Energy-Efficient Routing Protocol for wireless sensor networks. IEEE Radio Commun. 2005, 43, 8-13. [CrossRef]

27. Chai, J.; James, N.K.; Ngai, W.T. Application of decision-making techniques in supplier selection: A systematic review of literature. Expert Syst. Appl. 2013, 40, 3872-3885. [CrossRef]

28. Murata, T.; Ishibuchi, H. Performance evaluation of genetic algorithms for flowshop scheduling problems. In Proceedings of the First IEEE Conference on Evolutionary Computation, IEEE World Congress on Computational Intelligence, Orlando, FL, USA, 27-29 June 1994.

29. Ghiasi, S. Optimal Energy Aware Clustering in Sensor Networks. Sensors 2002, 2, 258-269. [CrossRef]

30. Shen, H. Finding the $\mathrm{k}$ Most Vital Edges with Respect to Minimum Spanning Tree. IEEE Aerosp. Electron. Conf. 1997, 1, 255-262. [CrossRef]

31. Dixit, S. Wireless Sensor Networks: Issues and Challenges. Int. J. Comput. Sci. Mob. Comput. 2014, 3, 681-685.

Publisher's Note: MDPI stays neutral with regard to jurisdictional claims in published maps and institutional affiliations.

(C) 2020 by the authors. Licensee MDPI, Basel, Switzerland. This article is an open access article distributed under the terms and conditions of the Creative Commons Attribution (CC BY) license (http://creativecommons.org/licenses/by/4.0/). 